\title{
Plantación de Celtis ehrenbergiana como técnica de recuperación de bosques invadidos por Ligustrum lucidum en los talares del NE de Buenos Aires
}

\author{
Maia C. Plaza Behr ${ }^{\varpi}$; Carolina A. Pérez; Juan F. Goya; Maximiliano Azcona \& \\ Marcelo F. Arturi
}

Laboratorio de Investigación de Sistemas Ecológicos y Ambientales, LISEA, Universidad Nacional de La Plata

\begin{abstract}
RESUMEN. El establecimiento de plantas exóticas en ecosistemas nativos puede disminuir la biodiversidad y provocar cambios funcionales en los sistemas invadidos. La reversión de esta situación puede requerir de la remoción de especies exóticas y la plantación de nativas. El ligustro (Ligustrum lucidum) es una especie arbórea invasora frecuente en bosques templados y subtropicales de Argentina. En los talares provoca una alta mortalidad de los árboles nativos sin que se observen procesos de regeneración que permitan la recuperación del bosque nativo. Realizamos ensayos de remoción de individuos adultos de ligustro originando dos claros de $500 \mathrm{~m}^{2}$ y dos de $200 \mathrm{~m}^{2}$. En los claros y en su periferia, bajo el dosel de ligustro, plantamos ejemplares de tala (Celtis ehrenbergiana) producidos en vivero y evaluamos su supervivencia y altura a los tres años. Observamos mayor altura media en las plantas ubicadas en el centro de los claros (68 \pm 3.3$)$ respecto de las del borde (52.6 \pm 1.7$)$ o bajo el dosel $(54.3 \pm 2.6)$. En los dos claros de $500 \mathrm{~m}^{2}$, los talas plantados presentaron menor altura media $(53.9 \pm 3$ y $66.5 \pm 4.9 \mathrm{~cm})$ que en los de $200 \mathrm{~m}^{2}(82.6 \pm 9.9$ y $91.3 \pm 8.5 \mathrm{~cm})$. Es posible que las plantas ubicadas en el centro de los claros hayan experimentado menor competencia por luz y recursos subterráneos que las ubicadas en los bordes o la periferia. A su vez, es probable que en los claros mayores, una combinación de una mayor competencia con plantas herbáceas, mayor exposición a la radiación y menor reparo haya resultado en un peor desarrollo del tala en comparación con los claros menores. Concluimos que el establecimiento del tala en talares invadidos es posible y sugerimos que el aprovechamiento de la madera del ligustro podría favorecer la aplicación de las técnicas ensayadas dado que presenta buenas propiedades para diversos usos.
\end{abstract}

[Palabras clave: especies exóticas, conservación, restauración ecológica]

\begin{abstract}
Aвstract. Celtis ehrenbergiana planting as a technique for the recovery of native forests invaded by Ligustrum lucidum in NE Buenos Aires. The establishment of alien plants in native ecosystems promotes biodiversity loss and impacts on ecological processes. Reverting that situation may require the remotion of alien species and the plantation of native species. Ligustrum lucidum is a common invasive tree in temperate and subtropical forests in Argentina. This species causes a high increase in the mortality of native trees in forests dominated by Celtis ehrenbergiana (talares). Native trees regeneration is almost null and then, there is no forest recovery. We carried out an experimental adult L. lucidum removal in two $500 \mathrm{~m}^{2}$ and two $200 \mathrm{~m}^{2}$ clearcuts. We planted C. ehrenbergiana produced in a greenhouse inside them, and in a peripheral area under the L. lucidum canopy. We observed higher mean height in the central area $(68 \pm 3.3)$ of the clearcuts than in the edge $(52.6 \pm 1.7)$ or the peripheral areas under the forest canopy $(54.3 \pm 2.6)$. Plants in $500 \mathrm{~m}^{2}$ clearcuts exhibited lower height $(53.9 \pm 3$ and $66.5 \pm 4.9 \mathrm{~cm}$ ) than those in $200 \mathrm{~m}^{2}$ clearcuts $(82.6 \pm 9.9$ and $91.3 \pm 8.5 \mathrm{~cm})$. Probably plants in the central area of the clearcuts were subjected to a weak competition, for light and underground resources, in comparison with plants in edges or peripheral areas. It is possible that in the larger clearcuts a higher competitive effect from herbaceous plants combined with higher radiation and lesser wind protection determined a poorer performance of planted C. ehrenbergiana than in the smaller ones. We conclude that the establishment of $C$. ehrenbergiana in forest invaded by L. lucidum is possible using the applied techniques. The use of the wood of the harvested L. lucidum, which has good properties for different applications, might make more viable the implementation of the techniques evaluated.
\end{abstract}

[Keywords: alien species, conservation, ecological restoration]

Editora asociada: Cingolani Ana

$\triangle$ mplazabehr@gmail.com
Recibido: 30 de septiembre de 2015

Aceptado: 15 de marzo de 2016 


\section{INTRODUCCIÓN}

El establecimiento de especies leñosas exóticas en bosques nativos es un proceso que ocurre en diferentes partes del mundo, y que en la mayoría de los casos se inicia a partir de la introducción intencional de especies (Richardson and Rejmánek 2011). En general, tiene un efecto negativo sobre la biodiversidad y produce profundos cambios en el funcionamiento de los ecosistemas invadidos (Dascanio et al. 1994; Aragón et al. 2014; Richardson et al. 2014).

Aunque aparentemente no existe un "síndrome invasor" (Petit et al. 2004), varios autores han coincidido en señalar las características de las especies leñosas con mayor potencial para convertirse en invasoras. Son de alto porte, con mecanismos eficientes de dispersión, capaces de reproducirse sexual y asexualmente, con una temprana madurez reproductiva, gran producción de semillas de pequeño tamaño, altas tasas de germinación y crecimiento relativo de los renovales y alta plasticidad fenotípica (Rejmánek and Richardson 1996; Alpert et al. 2000; Petit et al. 2004; Lamarque et al. 2011).

La especiearbórea asiática Ligustrumlucidum W.T. Aiton (ligustro) fue descripta como invasora en distintos bosques de nuestro país. Entre los más estudiados se encuentran los bosques montanos en las Yungas (Grau and Aragón 2000), los bosques serranos de Córdoba (Hoyos et al. 2010), la selva marginal de Punta Lara (Dascanio et al. 1994) y los talares del NE de Buenos Aires (Ribichich and Protomastro 1998). Es una especie de crecimiento rápido que fructifica y germina de manera muy abundante (Montaldo 1993). Las semillas pierden su capacidad para germinar al cabo de un año, por lo que no existe un banco de semillas (Montaldo 1993). Puede germinar y establecerse en un rango amplio de condiciones de cobertura arbórea, y crece más rápidamente que varias especies arbóreas nativas de los bosques subtropicales del NO de Argentina (Aragón and Groom 2003). Además de los eficientes procesos de reproducción sexual, el ligustro presenta una propagación vegetativa importante (Montaldo 1993) y es capaz de rebrotar de cepa. Su crecimiento en diámetro es rápido, y en los talares del NE de Buenos Aires alcanza entre 12 y $14 \mathrm{~m}$ de altura (Arturi, observación personal).

Los talares son bosques dominados por Celtis ehrenbergiana (Klotzsch) Liebm. var. ehrenbergiana (tala), por Scutia buxifolia
Reissek (coronillo), o bien codominados por ambas especies, y constituyen el principal sistema boscoso de la provincia de Buenos Aires (Goya et al. 1992). En algunos sectores el bosque presenta distinto grado de invasión por especies exóticas, principalmente ligustro (Ribichich and Protomastro 1998). En los talares, el ligustro se establece y alcanza el estrato superior en un tiempo relativamente corto (alrededor de 10 años), y genera una cobertura continua quesupera la altura máxima que alcanzan las especies nativas (10 a 12 m), lo que provoca una mortalidad elevada (Arturi, observación personal). La fructificación y germinación del tala son abundantes, pero se observa una mortalidad alta de plántulas. En consecuencia, su regeneración es escasa o nula, aun en bosques no invadidos y en los pastizales sobre cordones desmontados (Arturi 1997; Arturi and Goya 2004).

En muchos casos, para revertir el proceso de invasión se requiere de la remoción de los árboles exóticos y la plantación de árboles nativos (Holmes et al. 2000; D'Antonio and Meyerson 2002). El éxito de plantación de nativas requiere que las condiciones creadas por la corta de árboles exóticos sean apropiadas para el establecimiento de los individuos plantados. Se ha señalado que los efectos positivos de la remoción de especies invasoras han sido limitados debido a la escasa superficie de los ensayos realizados, a la reinstalación de la invasora preexistente $\mathrm{u}$ otras nuevas, y /o a la escasa recuperación de las especies nativas (Kettenring and Adams 2011). En el caso del ligustro no hay datos concluyentes sobre las técnicas de control (Maranta 2014) y no se conocen en nuestro país estudios que incluyan la reforestación con nativas en bosques invadidos por esta especie.

En los bosques del NE bonaerense, la regeneración de tala en bosques invadidos por ligustro es poco probable a menos que se realicen prácticas tendientes a su recuperación. Esto implica disminuir el efecto competitivo de la especie invasora sobre las nativas. Para estudiar esos procesos realizamos un ensayo de reforestación con tala en claros generados en bosques invadidos por ligustro. Planteamos como hipótesis que la supervivencia y crecimiento de los talas plantados es mayor en las áreas centrales de los claros que en los bordes y bajo el dosel debido a que en estas últimas posiciones existe mayor competencia con los árboles remanentes. Además, planteamos que el efecto de la posición 
respecto del claro sobre la supervivencia y crecimiento depende del tamaño del claro, que influye sobre las condiciones ambientales que experimentan las plantas en el área central. Este trabajo tuvo como objetivos analizar el efecto de la posición de las plantas de tala respecto del claro (bajo dosel, en borde del claro y en centro del claro) sobre su supervivencia y crecimiento y analizar el efecto del tamaño del claro sobre estas mismas variables.

\section{Materiales y Métodos}

\section{Área de estudio}

Los talares se extienden sobre cordones conchiles (relieve positivo) subparalelos a la costa del Río de la Plata, formando parches de extensión variable en una matriz de zonas bajas y anegables donde se desarrollan pastizales y pajonales (Goya et al. 1992; Arturi and Goya 2004). Debido a su biodiversidad, los talares han sido incluidos en el programa El Hombre y la Biosfera (MAB-UNESCO) con la creación del "Parque Costero del Sur". El principal uso que se les da es ganadero y minero (extracción de conchilla), y como actividades secundarias se desarrollan emprendimientos relacionados con el turismo rural. Las actividades extractivas principalmente y las ganaderas, en muchos casos, implican la pérdida de la cobertura boscosa sin que se observe la recuperación espontánea del bosque. Los sitios con regeneración espontánea de tala, como plantaciones de árboles exóticos o montículos de suelo removido por diferentes actividades humanas, presentan menor biomasa total de raíces en comparación con el bosque nativo y los pastizales (Arturi and Goya 2004). No se dispone de datos de crecimiento en altura de dichos renovales, pero ejemplares plantados en situaciones controladas pueden alcanzar unos $5 \mathrm{~m}$ de altura a los 10 años y comienzan a producir frutos a esa misma edad (Arturi, observación personal).

El estudio se realizó en la estancia El Destino, ubicada en la Reserva de Biosfera "Parque Costero del Sur", entre las localidades de Magdalena y Punta Indio (provincia de Buenos Aires). La temperatura media anual varía entre 15 y $17{ }^{\circ} \mathrm{C}$, y la precipitación media anual oscila entre 800 y 1000 mm, con una evapotranspiración potencial anual entre 700 y $800 \mathrm{~mm}$. En promedio, esto determina un exceso de agua en el balance anual; sin embargo, a lo largo del año puede ocurrir una alternancia de períodos húmedos y de sequías fuertes (Burgos 1968; Arturi 1997). Los cordones de conchilla sobre los que se encuentran los parches de bosque fueron producto de diferentes ingresiones marinas del Cuaternario (Tricart 1973). Estos cordones se elevan entre 1 y $2 \mathrm{~m}$ de altura respecto del nivel general del terreno y presentan suelos bien drenados, cuyo perfil está poco desarrollado, con un horizonte A1 de $20 \mathrm{~cm}$ de profundidad, de textura franca con presencia de fragmentos gruesos de conchilla (Sánchez et al. 1976; Hurtado and Ferrer 1988).

\section{Instalación del ensayo}

Seleccionamos rodales formados originalmente por tala y coronillo que en la actualidad se encuentran en un estado avanzado de colonización por ligustro. En estos sitios la densidad relativa de ligustro varía entre 60 y 80\%, y el porcentaje de árboles nativos muertos entre 40 y $85 \%$ respecto del total de árboles nativos presentes (Arturi and Goya, datos no publicados). En estos sitios generamos cuatro claros circulares, dos de 500 $\mathrm{m}^{2}$ y dos de $200 \mathrm{~m}^{2}$. El tamaño del claro fue asignado aleatoriamente a cada sitio. En agosto de 2010 apeamos la totalidad de los ligustros con motosierra y retiramos los residuos de la corta y los árboles nativos muertos. El número de árboles nativos vivos varió entre uno y tres por claro, encontrándose en todos los casos desprovistos de copa desarrollada debido al efecto competitivo del ligustro. Los claros se localizaron en tres cordones diferentes con una distancia mínima de 70 m y máxima de 120 $\mathrm{m}$ entre ellos. Realizamos la plantación en los dos meses posteriores a la formación de los claros (septiembre-octubre de 2010) utilizando un distanciamiento de $3 \times 3 \mathrm{~m}$. Distinguimos tres posiciones respecto del claro: dosel (en la periferia del claro, bajo el dosel del bosque), borde de claro (cobertura parcial del bosque) y área central del claro (sin cobertura del bosque). Las plantas del tratamiento dosel, ubicadas entre 2 y $5 \mathrm{~m}$ desde el borde del claro hacia el interior del bosque invadido, fueron consideradas como testigo. En total, en los cuatro claros y las tres posiciones, plantamos 206 talas (Tabla 1). Los mismos fueron producidos en la Unidad de Vivero Forestal de la Facultad de Ciencias Agrarias y Forestales a partir de semillas colectadas en la zona de estudio y cultivados en macetas de $3 \mathrm{dm}^{3}$. Al momento de ser plantadas, las plantas de tala tenían 2 años de edad y una altura promedio \pm error de la media de $55.2 \pm 1$ $\mathrm{cm}$, y las asignamos aleatoriamente a los 
distintos claros y posiciones. Un año después de la instalación del ensayo (octubre de 2011) realizamos un único desmalezado con motoguadaña eliminando plantas herbáceas y rebrotes de ligustro. No aplicamos ningún otro tipo de tratamiento. Hasta diciembre de 2013 determinamos anualmente la supervivencia y registramos la altura de las plantas vivas. Observamos incrementos y disminuciones de la altura debidos al crecimiento y a la mortalidad de porciones apicales del tallo, respectivamente. Como indicador del balance entre ambos procesos consideramos para el análisis la altura alcanzada por las plantas vivas en diciembre de 2013.

Tabla 1. Número de plantas de C. ehrenbergiana plantadas en cada posición respecto del claro y en cada uno de los tamaños de claro.

Table 1. Number of $C$. ehrenbergiana plants planted per position and clearcut size.

\begin{tabular}{lcccc}
\hline Posición & Claro de & \multicolumn{3}{c}{ Número de plantas de } \\
& $500 \mathrm{~m}^{2}$ & $500 \mathrm{~m}^{2}$ & $200 \mathrm{~m}^{2}$ & $200 \mathrm{~m}^{2}$ \\
\hline Dosel & 18 & 13 & 11 & 19 \\
$\begin{array}{l}\text { Borde de } \\
\text { claro }\end{array}$ & 19 & 27 & 12 & 4 \\
$\begin{array}{l}\text { Centro de } \\
\text { claro }\end{array}$ & 30 & 33 & 8 & 12 \\
\hline
\end{tabular}

\section{Análisis estadístico}

El diseño del experimento resultó asimilable al de "parcela dividida" con un tratamiento a nivel de parcela ("tamaño del claro") y otro a nivel de subparcela ("posición respecto del claro"). Consideramos a los claros como unidades experimentales de manera que los seis tratamientos resultantes de la combinación de los factores "tamaño del claro" por "posición respecto del claro" contó con dos repeticiones. En el contexto de los modelos mixtos, lineales y no lineales, estos experimentos pueden analizarse mediante la inclusión de un factor aleatorio que representa cada una de las parcelas (Faraway 2006). Los análisis estadísticos se realizaron mediante el programa de uso libre R. La comparación de los modelos que incluyen o excluyen el factor aleatorio la llevamos a cabo siguiendo el procedimiento descripto por Faraway (2006), tanto para el análisis de la supervivencia como para la altura.

Para estimar el efecto del tamaño del claro y la posición respecto del claro sobre la supervivencia, realizamos una regresión logística. Utilizamos el paquete lme4 (Bates et al. 2015). Se ingresaron dos columnas indicando el número de plantas vivas y muertas respectivamente, para cada uno de los seis tratamientos con sus dos repeticiones. Se utilizaron otras tres columnas para indicar mediante variables categóricas la clase de tamaño del claro, la posición respecto del claro y la identidad del claro (1 a 4) que representó el factor aleatorio. Se llevó a cabo un ajuste secuencial desde el modelo nulo con 12-1 grados de libertad (g.1.), ingresando luego el tamaño del claro (g.l.=1), la posición respecto del claro (g.l.=2) y, por último, su interacción (g.l.=2). De esta manera, los grados de libertad del denominador en el cálculo de la Deviance, calculados como la diferencia entre el modelo ajustado y el modelo nulo, fueron respectivamente 10,8 y 6 . Se ajustó un modelo mixto para evaluar la significancia del factor aleatorio que identifica a cada claro como individuo sobre el cual se realizaron las observaciones.

Estimamos el efecto de los tratamientos sobre la altura media de las plantas mediante un modelo lineal mixto. La posición respecto del claroy su categoría de tamaño representaronlos factores fijos y el claro (1 a 4) el factor aleatorio. El valor ingresado en los análisis fue la media de la altura de las plantas en cada posición, en cada claro. De esta manera dispusimos de dos repeticiones para cada tratamiento. El total de observaciones fue 12 y los grados de libertad del error en las pruebas de los efectos principales y su interacción fue 6. Analizamos los datos con el paquete nlme (Pinheiro et al. 2015), siguiendo el procedimiento descripto por Faraway (2006).

\section{Resultados}

La posición de las plantas de tala respecto del claro presentó un efecto significativo sobre la supervivencia (Deviance ${ }_{2,8}=9.2, P<0.05$ ). Observamos mayor supervivencia en las áreas centrales de los claros que en el resto de las posiciones evaluadas (Figura 1). El efecto del tamaño del claro sobre la supervivencia no resultó significativo, pero observamos una tendencia (Deviance (D,10 $_{10}=2.4, P=0.12$ ) a mayor supervivencia en los claros de $200 \mathrm{~m}^{2}$ (41\%) que en los de $500 \mathrm{~m}^{2}$ (34\%). La interacción entre ambos factores no resultó significativa (Deviance $_{2,6}=3.8, P=0.15$ ).

La altura media de las plantas fue afectada significativamente por su posición dentro del claro $\left(F_{26}=9.4, P<0.05\right)$ y por el tamaño del mismo $\left(F_{1,6}=15.6, P<0.01\right)$. Las plantas en las 


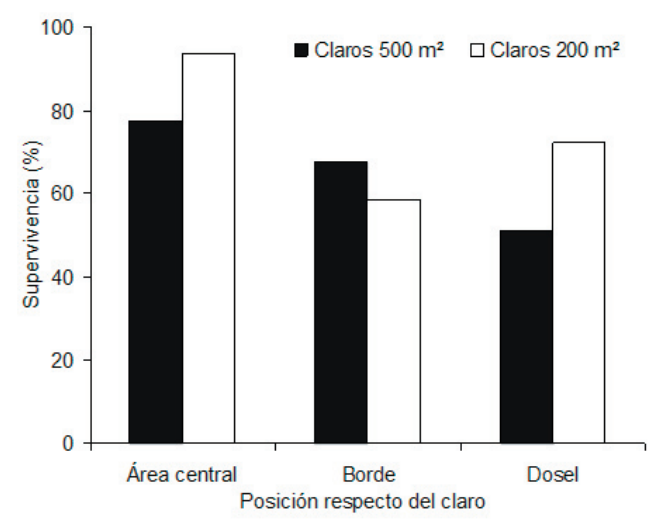

Figura 1. Porcentaje de plantas de C. ehrenbergiana vivas (supervivencia) en cada posición y tamaño de los claros.

Figure 1. Percent of live $C$. ehrenbergiana plants per position and clearcut size.

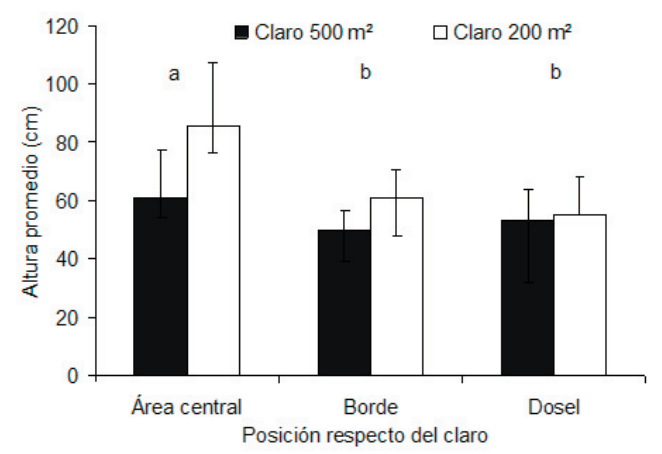

Figura2. Alturapromediodelasplantasde C.ehrenbergiana para cada posición y cada tamaño de claro. Se indica la media \pm error de la media, letras distintas indican diferencias significativas entre posiciones $(P<0.05)$.

Figure 2. Mean height of $C$. ehrenbergiana plants per position and clearcut size. Mean \pm mean error are indicated, significant differences between positions $(P<0.05)$.

áreas centrales de los claros presentaron mayor altura media $(68.0 \pm 3.3)$ que las plantas en el borde del claro $(52.6 \pm 1.7)$ y debajo del dosel (54.3 \pm 2.6$)$. A su vez las alturas medias fueron mayores en los claros de $200 \mathrm{~m}^{2}(82.6 \pm 9.9 \mathrm{y}$ $91.3 \pm 8.5 \mathrm{~cm})$ que en los de $500 \mathrm{~m}^{2}(53.9 \pm 3$ y $66.5 \pm 4.9 \mathrm{~cm}$ ) (Figura 2). La interacción entre ambos factores no resultó significativa $\left(F_{2,6}=2.6, P=0.16\right)$. El factor aleatorio "claro" no fue significativo ( $P>0.05)$ en ninguno de los análisis realizados.

\section{DisCUSIÓN}

La remoción de ligustros adultos tuvo un efecto positivo sobre la supervivencia y el crecimiento de plantas de tala. La mayor supervivencia y crecimiento de las plantas ubicadas en áreas centrales de los claros respecto de las ubicadas en el borde o bajo el dosel podrían vincularse con el aumento en la disponibilidad de luz y la disminución de la competencia por recursos del suelo. La disponibilidad de luz, como un factor clave en el establecimiento de plántulas, fue reconocida en muchos bosques del mundo (Gerhardt 1996; Lewis and Tanner 2000; Kern et al. 2014). También se ha demostrado que la competencia subterránea tiene marcada influencia en la supervivencia y crecimiento de renovales en diferentes bosques (Coomes and Grubb 2000) y en particular en los talares (Arturi 1997; Arturi and Goya 2004). En las plantaciones de árboles exóticos (pino y eucalipto), los renovales de tala tienden a responder de manera positiva a una mayor disponibilidad de luz determinada por discontinuidades en la cobertura del dosel. Mientras tanto, en el bosque nativo, con mayor competencia subterránea, los pocos renovales encontrados tendieron a ubicarse en los sitios más sombreados (Arturi and Goya 2004). Esto indicaría que las plantas sometidas a mayor competencia subterránea responden de forma negativa al aumento en los niveles de radiación. Dado que cuando se plantaron los talas, los claros se encontraban prácticamente desprovistos de vegetación arbórea y herbácea, las plantas establecidas bajo el dosel o en el borde posiblemente experimentaron el efecto competitivo ejercido por los árboles adultos, resultando en un menor crecimiento y supervivencia que las ubicadas en áreas centrales de los claros. La reducción de la cobertura arbórea en los talares promueve un aumento de la expansión de herbáceas anuales de ciclo invernal (Carduus acanthoides L., Carduus pycnocephalus L.) o estival (Solidago chilensis Meyen) que pueden alcanzar coberturas superiores a 75\% (Arturi et al. 2010). Si bien en el presente ensayo no se realizaron estimaciones de la cobertura o biomasa herbácea, se observó un mayor desarrollo de las mismas en los claros de 500 $\mathrm{m}^{2}$ que en los de $200 \mathrm{~m}^{2}$. Es probable que esa vegetación herbácea haya provocado un efecto competitivo con consecuencias negativas sobre el crecimiento, y probablemente la supervivencia, de los talas plantados, en especial en las áreas centrales. Los resultados encontrados indican que en el período de tres años analizado, dicho efecto no presentó la magnitud suficiente como para anular el efecto positivo de la disminución de la competencia con los ligustros adultos. Sin embargo, en los claros de $500 \mathrm{~m}^{2}$, los talas plantados presentaron un crecimiento menor (y una tendencia a sobrevivir menos) que en los de 200 $\mathrm{m}^{2}$, en concomitancia con el mayor desarrollo 
herbáceo observado, y probablemente mayor exposición a condiciones de desecación. Tanto para la supervivencia como para el crecimiento, los gráficos permiten observar que las diferencias entre los centros de los claros y las áreas bajo dosel son mayores en los claros de $200 \mathrm{~m}^{2}$ que en los de $500 \mathrm{~m}^{2}$. Esas relaciones sugieren que los resultados tienden a coincidir con la hipótesis planteada: la posición respecto del claro presenta un efecto dependiente del tamaño del mismo. El resultado más destacable es que el centro de los claros de $200 \mathrm{~m}^{2}$ presenta mejores condiciones para el establecimiento y crecimiento de tala que el centro de los claros de $500 \mathrm{~m}^{2}$. Sin embargo, algunas de esas tendencias no resultaron significativas, como el efecto del "tamaño del claro" sobre la supervivencia y la interacción entre los factores "tamaño del claro" y "posición respecto del claro" en ambas variables. Es posible que la falta de significancia esté relacionada con la baja potencia del experimento, debido a que presentó sólo dos repeticiones y, en el caso de la supervivencia, el número de plantas disponibles para el cálculo de esas tasas resultó reducido.

Muchos estudios que ensayaron sólo la remoción de las especies exóticas presentaron escaso éxito en la restauración de ambientes invadidos (Kettenring and Adams 2011). En nuestro trabajo, si bien no se cuantificaron los procesos de rebrote de los ligustros cortados ni el establecimiento de nuevos individuos por germinación, se observó un alto porcentaje de rebrote (60 a $100 \mathrm{~cm}$ de altura) y muy bajo establecimiento de renovales por semillas, tanto de árboles nativos como de exóticos. Por lo tanto, la recuperación de los talares sería efectiva sólo si se aplica en conjunto la remoción de ligustro con la plantación de especies arbóreas nativas y se efectúa un control del rebrote de los ligustros durante los primeros años.

Los resultados encontrados sugieren que la erradicación total del ligustro en los talares podría representar un objetivo poco realista en virtud del esfuerzo requerido por los tratamientos aplicados. En cambio, resulta posible planificar actividades tendientes a conservar las áreas menos afectadas y desarrollar un proceso paulatino de restauración de las áreas más invadidas. La madera del ligustro presenta buenas propiedades para diversos usos (Benetti 2015) y su aprovechamiento podría significar una posible fuente de recursos para la zona, haciendo más factible la aplicación de las técnicas ensayadas.

Agradecimientos. Este trabajo fue financiado por la Facultad de Ciencias Agrarias y Forestales de la UNLP. Agradecemos a la Fundación Elsa Shaw de Pearson por apoyar la realización de estos ensayos en la Estancia El Destino, a la Unidad de Vivero Forestal de la FCAyF y a los estudiantes de Ingeniería Forestal que colaboraron con los trabajos de campo. También agradecemos a los revisores anónimos del trabajo y a la Dra. Cingolani por sus valiosos comentarios y sugerencias.

\section{REFERENCIAS}

Alpert, P., E. Bone, and C. Holzapfel. 2000. Invasiveness, invasibility and the role of environmental stress in the spread of non-native plants. Perspectives in plant ecology, evolution and systematic 3(1):52-66.

Aragón, R., and M. Groom. 2003. Invasion by Ligustrum lucidum (Oleaceae) in NW Argentina: early stage characteristics in different habitat types. International Journal of Tropical Biology and Conservation 51(1):59-70.

Aragón, R., L. Montti, M. M. Ayup, and R. Fernández. 2014. Exotic species as modifiers of ecosystem processes: Litter decomposition in native and invaded secondary forests of NW Argentina. Acta Oecologica 54:21-28.

Arturi, M. F. 1997. Regeneración de Celtis tala y su relación con el pastoreo, la cobertura herbácea y arbórea en el NE de la provincia de Buenos Aires, Argentina. Ecología Austral 7:3-12.

Arturi, M. F., C. Pérez, and S. Torres Robles. 2010. Plant traits and canopy types: seasonal and local variation in a grazed semi-deciduous temperate woodland. Phytocoenologia 40(4):245-253.

Arturi, M. F., and J. F. Goya. 2004. Estructura, dinámica y manejo de los talares del NE de Buenos Aires [Capítulo 10]. Pp. 1-23 en: M. F. Arturi, J. L. Frangi y J. F. Goya (eds.). Ecología y manejo de los bosques de Argentina. Publicación multimedia. Editorial de la Universidad Nacional de La Plata. Buenos Aires, Argentina.

Bates, D., M. Maechler, B. Bolker, and S. Walker. 2015. lme4: Linear mixed-effects models using Eigen and S4. R package version 1.1-9. En: https://CRAN.R-project.org/package=lme4.

Benetti, F. 2015. Estudio de la durabilidad natural y de las propiedades tecnológicas de la madera de Ligustrum lucidum W.T. Aiton. Tesis de grado. Universidad Nacional de La Plata.

Burgos, J. J. 1968. El clima de la Provincia de Buenos Aires. Pp. 33-101 en: A. L. Cabrera (ed.). Flora de la Provincia de Buenos Aires. Colección Científica del INTA. Buenos Aires, Argentina.

Coomes, D. A., and P. J. Grubb. 2000. Impacts of root competition in forests and woodlands: a theoretical framework and review of experiments. Ecological monographs 70(2):171-207. 
D'Antonio, C., and L. A. Meyerson. 2002. Exotic plant species as problems and solutions in ecological restoration: a synthesis. Restoration Ecology 10(4):703-713.

Dascanio, L. M., M. D. Barrera, and J. L. Frangi. 1994. Biomass structure and dry matter dynamics of subtropical alluvial and exotic Ligustrum forests at the Río de la Plata, Argentina. Vegetatio 115(1):61-76.

Faraway, J. J. 2006. Extending the linear model with r: generalized linear, mixed effects and nonparametric regression models. Chapman and Hall. Florida, EE UU.

Gerhardt, K. 1996. Effects of root competition and canopy openness on survival and growth of tree seedlings in a tropical seasonal dry forest. Forest Ecology and Management 82(1):33-48.

Goya, J. F., G. Placci, M. F. Arturi, and A. Brown. 1992. Distribución y características estructurales de los Talares de la Reserva de Biosfera Parque Costero del Sur. Revista de la Facultad de Agronomía, La Plata 68(1):53-64.

Grau, H. R., and R. Aragón. 2000. Árboles invasores de la sierra de San Javier. Pp. 5-20 en: HR Grau and R Aragón (eds.). Arboles exóticos de las Yungas argentinas. LIEY, Universidad Nacional de Tucumán, Argentina.

Holmes, P. M., D. M. Richardson, B. W. Van Wilgen, and C. Gelderblom. 2000. Recovery of South African fynbos vegetation following alien woody plant clearing and fire: implications for restoration. Austral Ecology 25:631-639.

Hoyos, L. E., G. I. Gavier-Pizarro, T. Kuemmerle, E. H. Bucher, V. C. Radeloff, and P. A. Tecco. 2010. Invasion of glossy privet (Ligustrum lucidum) and native forest loss in the Sierras Chicas of Córdoba, Argentina. Biological invasions 12(9):3261-3275.

Hurtado, M., and J. A. Ferrer. 1988. Guía de Campo de las Segundas Jornadas de Suelos de la Región Pampeana. La Plata, Argentina.

Kettenring, K. M., and C. R. Adams. 2011. Lessons learned from invasive plant control experiments: a systematic review and meta analysis. Journal of Applied Ecology 48(4):970-979.

Kern, C. C., R. A. Montgomery, P. B. Reich, and T. F. Strong. 2014. Harvest-created canopy gaps increase species and functional trait diversity of the forest ground-layer community. Forest Science 60(2):335-344.

Lamarque, L. J., S. Delzon and C. J. Lortie. 2011. Tree invasions: a comparative test of the dominant hypotheses and functional traits. Biological Invasions 13(9):1969-1989.

Lewis, S. L., and E. V. Tanner. 2000. Effects of above-and belowground competition on growth and survival of rain forest tree seedlings. Ecology 81(9):2525-2538.

Maranta, A. 2014. Control de leñosas exóticas en reservas naturales. En: http://www.habitatydesarrollo.org.ar/ imagenes2014/InformeDiagnosticoExoticas.pdf

Montaldo, N. H. 1993. Dispersión por aves y éxito reproductivo de dos especies de Ligustrum (Oleaceae) en un relicto de selva subtropical en la Argentina. Revista Chilena de Historia Natural 66:75-85.

Petit, R. J., R. Bialozyt, P. Garnier-Géré, and A. Hampe. 2004. Ecology and genetics of tree invasions: from recent introductions to Quaternary migrations. Forest Ecology and Management 197(1):117-137.

Pinheiro, J., D. Bates, S. DebRoy, D. Sarkar, and R Core Team. 2015. nlme: Linear and Nonlinear Mixed Effects Models. R package version 3.1-122. En: http://CRAN.R-project.org/package=nlme.

Rejmánek, M., and D. M. Richardson. 1996. What attributes make some plant species more invasive? Ecology 77(6): 1655-1661.

Ribichich, A. M., and J. Protomastro. 1998. Woody vegetation structure of xeric forest stands under different edaphic site conditions and disturbance histories in the Biosphere Reserve ,Parque Costero del Sur', Argentina. Plant Ecology 139(2):189-201.

Richardson, D. M., and M. Rejmánek. 2011. Trees and shrubs as invasive alien species - a global review. Diversity Distrib. 17(5):788-809.

Richardson, D. M., C. Hui, M. A. Núñez, and A. Pauchard. 2014. Tree invasions: patterns, processes, challenges and opportunities. Biological invasions 16(3):473-481.

Sánchez, R. O., J. A. Ferrer, O. A. Duymovich, and M. A. Hurtado. 1976. Estudio pedológico integral de los Partidos de Magdalena y Brandsen (Provincia de Buenos Aires). Anales del LEMIT Serie II N 310, Ministerio de Obras Públicas de la Provincia de Buenos Aires.

Tricart, J. 1973. Geomorfología de la Pampa Deprimida: base para los estudios edafológicos y agronómicos. Plan Mapa de Suelos de la Región Pampeana. XII Colección Científica del INTA. Buenos Aires. 